M. Stöhr, R. Sadanandan and W. Meier, Experimental study of unsteady flame structures of an oscillating swirl flame in a gas turbine model combustor, Proceedings of the Combustion Institute 32 (2009) 2925-2932.

The original publication is available at www.elsevier.com

http://dx.doi.org/10.1016/j.proci.2008.05.086 


\title{
Experimental study of unsteady flame structures of an oscillating swirl flame in a gas turbine model combustor
}

\author{
M. Stöhr *, R. Sadanandan, W. Meier \\ German Aerospace Center (DLR), Institute of Combustion Technology, \\ Pfaffenwaldring 38-40, 70569 Stuttgart, Germany
}

\begin{abstract}
Velocity fields and flame structures of a partially premixed swirl flame in a gas turbine model combustor are measured in axial and transverse sections using simultaneous (Stereo-)PIV and OH-PLIF. The flame, operated under atmospheric pressure with air and methane at a thermal power of $10.3 \mathrm{~kW}$ and a global equivalence ratio of $\phi=0.75$, features thermoacoustic oscillations at a frequency $f \approx 295$ Hz. The averaged flow field with inner and outer recirculation zones is typical of swirl-stabilized flames, and the instantaneous measurements show the presence of a helical vortex (PVC) located in the inner shear layer. The PVC, which rotates with a different frequency than the thermoacoustic oscillation, leads to an enhanced mixing of burned and unburned gas and thus to stabilization of the flame. Two distinct large-scale structures of velocity and $\mathrm{OH}$ are found in the transverse cross-sections. The first type is characterized by a roughly annular region of inflowing unburned gas and distinct inner and outer recirculation zones. In the second type, the region of positive axial velocity forms a spiral, and the recirculation zone consists of an inner region that is connected to the outer parts by a narrow curved zone along the spiral arm. Whereas the first type is a typical scenario of vortex breakdown with a PVC, the transient spiral recirculation zone observed here has, to our knowledge, not yet been reported. A phase-resolved analysis shows that the annular form correlates with low, and the spiral form with high rates of global heat release.
\end{abstract}

Key words: Gas turbine combustion, Vortex breakdown, Simultaneous PIV and OH-PLIF, Thermoacoustic oscillation

* Corresponding author. Fax: +497116862578, Email: michael.stoehr@dlr.de 


\section{Introduction}

Modern gas turbine (GT) combustors are operated under lean premixed or partially premixed conditions in order to achieve low emissions of $\mathrm{NO}_{x}$. Flames are most often stabilized by inducing a swirling flow of the reactants, which leads to an inner recirculation of hot burned gases and thereby enhances ignition of unburned gas. However, the operation of GT combustors under such conditions is highly susceptible to thermoacoustic oscillations, which may strongly affect lifetime and reliability of the GT. The mechanisms of these oscillations are still not well understood, and their prediction is a critical issue of combustor design.

The formation of the inner recirculation zone (IRZ) results from vortex breakdown of the swirling flow [1-3]. An important aspect of this phenomenon is the occurence of large-scale coherent structures such as the precessing vortex core (PVC). The PVC is an unsteady vortex located in the shear layer of the IRZ that precesses around the central axis. Several experimental works have found PVCs in isothermal flows in GT combustors for swirl numbers $S>0.5$ [4-7]. The occurence and role of PVCs under combustion conditions is a complex issue and strongly depends on mode of fuel entry, equivalence ratio and combustor geometry [8]. Only few experimental studies have addressed the interaction of flame and PVC, and the mechanisms are largely unclear. Schildmacher and Koch reported the presence of a PVC under isothermal conditions which disappeared for the reacting case with same flow conditions [9]. Syred et al. found a PVC under reacting conditions and discussed its effect of increased mixing [10]. Li and Gutmark proposed that a PVC is the main factor driving the combustion instability in a swirl-dump combustor [11].

Strong efforts are currently undertaken for the numerical simulation of swirlstablized flames with the intentional use for the design of improved GT combustors. Numerous recent studies have shown that large eddy simulation (LES) is capable of reproducing unsteady swirl-induced vortex structures like the PVC for both isothermal [12-14] and reacting [15-23] cases. Some of these studies found a PVC in the isothermal flow that disappeared under reacting conditions $[15,20,22]$. Duwig and Fuchs found a PVC in a swirl-stabilized flame for $S=1.05$ and $S=1.35$, but not for $S=0.45$ [19].

The present work investigates the interaction between a PVC and a GTtypical swirl flame by means of planar laser diagnostics. Velocity fields and flame structures in axial and transverse sections were simultaneously measured using particle image velocimetry (PIV) and planar laser-induced fluorescence (PLIF) of $\mathrm{OH}$. In a recent study we discussed the potentials and limitations of this experimental technique, and reviewed its application by other groups [24]. The flame is operated with $\mathrm{CH}_{4}$ and air in a GT model combustor under 
atmospheric pressure. It has a thermal power of $10.3 \mathrm{~kW}$, a global equivalence ratio of $\phi=0.75$ and a swirl number of $S \approx 0.55$. Furthermore it features thermoacoustic oscillations at $f \approx 295 \mathrm{~Hz}$. Several experimental studies have been applied to the same combustor and operating condition: distributions of $\mathrm{OH}$, $\mathrm{CH}$ and $\mathrm{H}_{2} \mathrm{CO}$ have been measured by PLIF $[24,25,27,28]$, major species concentrations have been determined using Raman spectroscopy [26,27,29] and velocites by laser Doppler velocimetry (LDV) and PIV [26-28,24]. The focus of this study is on the phenomenology of unsteady large-scale coherent structures in a GT typical swirl flame. A PVC is identfied in instantaneous velocity fields and its effect on flame structure is discussed using the corresponding OH-PLIF images. Furthermore large-scale structural changes of flow field and reaction zone during a cycle of the thermoacoustic oscillation are characterized. The observed phenomena are compared to phase-dependent variations of heat release and pressure in the combustion chamber.

\section{Experimental methods}

\subsection{Combustor and flame}

A schematic of the gas turbine model combustor is shown in fig. 1. Dry air at room temperature enters a plenum (diameter $79 \mathrm{~mm}$, height $65 \mathrm{~mm}$ ) and then separately passes two radial swirl generators. The two co-swirling flows enter the combustion chamber through a central nozzle (diameter $15 \mathrm{~mm}$ ) and an annular nozzle (inner diameter $17 \mathrm{~mm}$, outer diameter $25 \mathrm{~mm}$ contoured to an outer diameter of $40 \mathrm{~mm}$ ). Non-swirling $\mathrm{CH}_{4}$ is fed through 72 channels

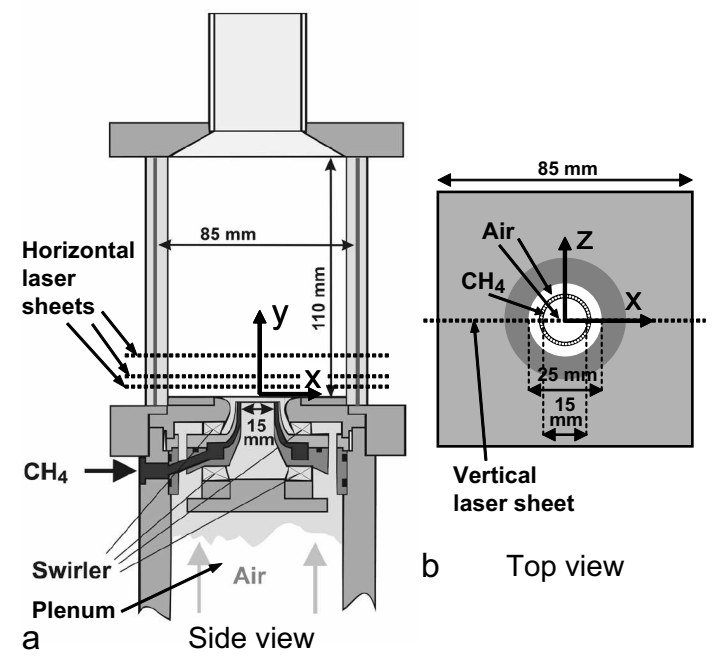

Fig. 1. (a) Side view of the gas turbine model combustor and (b) top view of burner exit plane. 
$\left(0.5 \times 0.5 \mathrm{~mm}^{2}\right)$ forming a ring between the air nozzles. The exit planes of the fuel and central air nozzles are located $4.5 \mathrm{~mm}$ below the exit plane of the outer air nozzle which is taken as height $y=0$. The combustion chamber has a square cross section of $85 \times 85 \mathrm{~mm}^{2}$, a height of $114 \mathrm{~mm}$, and is enclosed by 4 quartz windows held by 4 posts (diameter $10 \mathrm{~mm}$ ) in the corners allowing very good optical access. A conical top plate with a central exhaust tube (diameter $40 \mathrm{~mm}$, length $50 \mathrm{~mm}$ ) forms the exit. In the present study the flame was operated with an air flow rate of $280.8 \mathrm{~g} / \mathrm{min}$ and $\mathrm{CH}_{4}$ flow rate of $12.3 \mathrm{~g} / \mathrm{min}$. This results in a thermal power $P_{\text {thermal }}=10.3 \mathrm{~kW}$ and a global equivalence ratio $\phi_{\text {global }}=0.75$. From the velocity profile at $y=1 \mathrm{~mm}$ obtained with LDV the swirl number, calculated as the ratio between the tangential and axial momentum flux, was estimated as $\mathrm{S} \approx 0.55$, and the Reynolds number of the cold inflow as Re $\approx 15000$ [29]. Under these conditions the flame exhibits thermoacoustic emissions at $f \approx 295 \mathrm{~Hz}$ with a total sound pressure level in the combustion chamber of $\approx 130 \mathrm{~dB}$. The pressure fluctuations were measured by two microphones (Brüel \& Kjaer), one attached to a post in the corner of the combustion chamber at $y=16 \mathrm{~mm}$, and the other at half height of the plenum wall.

\subsection{Laser diagnostics}

This study employs a diagnostic setup that has for the most part been detailed in a recent publication [24], and therefore we will describe it rather briefly. The setup consists of two separate laser and camera systems used for simultaneous PIV and OH-PLIF measurements. The beams of the two laser systems are expanded into two coplanar light sheets crossing the combustion chamber. The setup was used in two versions with different orientations of the light sheets: first, a vertical section through the central axis ( $z=0$, shown in fig. 1b), and second, horizontal transverse sections located at $y=5,10$ and $20 \mathrm{~mm}$ (shown in fig. 1a). The former case using two-component PIV is mostly equivalent to the setup used in [24]. For the latter configuration, the PIV system (LaVision Flowmaster) was extended to a stereoscopic setup as shown in fig. 2, where both the two PIV cameras and the LIF camera are located diagonally above the laser sheet. For both configurations the rate of aquisition was 5 fps. The absolute measuring uncertainty of instantaneous velocities was estimated as $\Delta v=0.6 \mathrm{~m} / \mathrm{s} . \mathrm{OH}^{*}$-chemiluminescence (integrated along the line-of-sight) was measured using the OH-PLIF detection system for the vertical section. Using a multichannel A/D converter, the microphone signals and the Q-switch trigger signal were continuously recorded during a series of measurements. This allows the determination of the phase-angle $\varphi$ of the thermoacoustic oscillation for each individual measurement. Following the definition used in $[25,26], \varphi=0^{\circ}$ corresponds to the minimum of pressure in the plenum, $\varphi=90^{\circ}$ to the negativeto-positive zero transition, etc. 


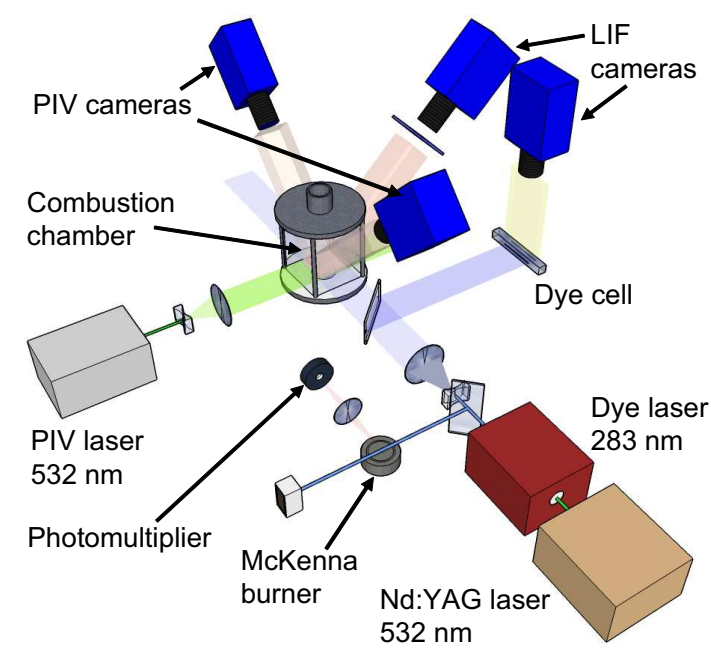

Fig. 2. Setup of simultaneous OH-PLIF and stereoscopic PIV diagnostics.

\section{Averages of flow field and reaction zone}

Figure 3 shows averaged fields of $\mathrm{OH}$ and velocity measured in the vertical section and $\mathrm{OH}^{*}$-chemiluminescence at 4 phase-angles $\varphi$. The velocity fields are typical of confined swirl flames, including a cone-shaped inflow of fresh gas at the nozzle exit, an inner recirculation zone (IRZ) and an outer recirculation zone (ORZ). A shear layer with strong velocity gradients is located between the IRZ and the inflowing stream, and a second shear layer is formed between the inflow and the ORZ. The velocity of inflowing unburned gas and the size of the IRZ show considerable variations with phase-angle.

$\mathrm{OH}^{*}$-chemiluminescence (spontaneous emission of electronically excited $\mathrm{OH}$ ) provides information about the location of heat release. The $\mathrm{OH}^{*}$ images show that the heat release zone starts at the height of $2-5 \mathrm{~mm}$ and extends up to $y \approx 35 \mathrm{~mm}$. The flame base therefore lies approximately $8 \mathrm{~mm}$ above the fuel nozzle exit, which implies that a high level of mixing has occured before ignition and that the flame is of partially premixed nature. Deconvolution of the integrated images (assuming axial symmetry) shows the heat release zone has a conical shape [24]. Both the total heat release and the radial extension of the flame change during the oscillation cycle.

Interpretation of OH-PLIF images is somewhat difficult due to the slow equilibration of $\mathrm{OH}$ after its formation, and we discuss this issue in the context of instantaneous OH-PLIF images in section 4. The averaged $\mathrm{OH}$ images show regions without $\mathrm{OH}$ (black) in the unburned gas directly above the burner nozzle, a cone-shaped area of high $\mathrm{OH}$ concentrations (white) near the reaction zones and low $\mathrm{OH}$ levels (gray) representing burned gas that has been transported away from the reaction zone. 


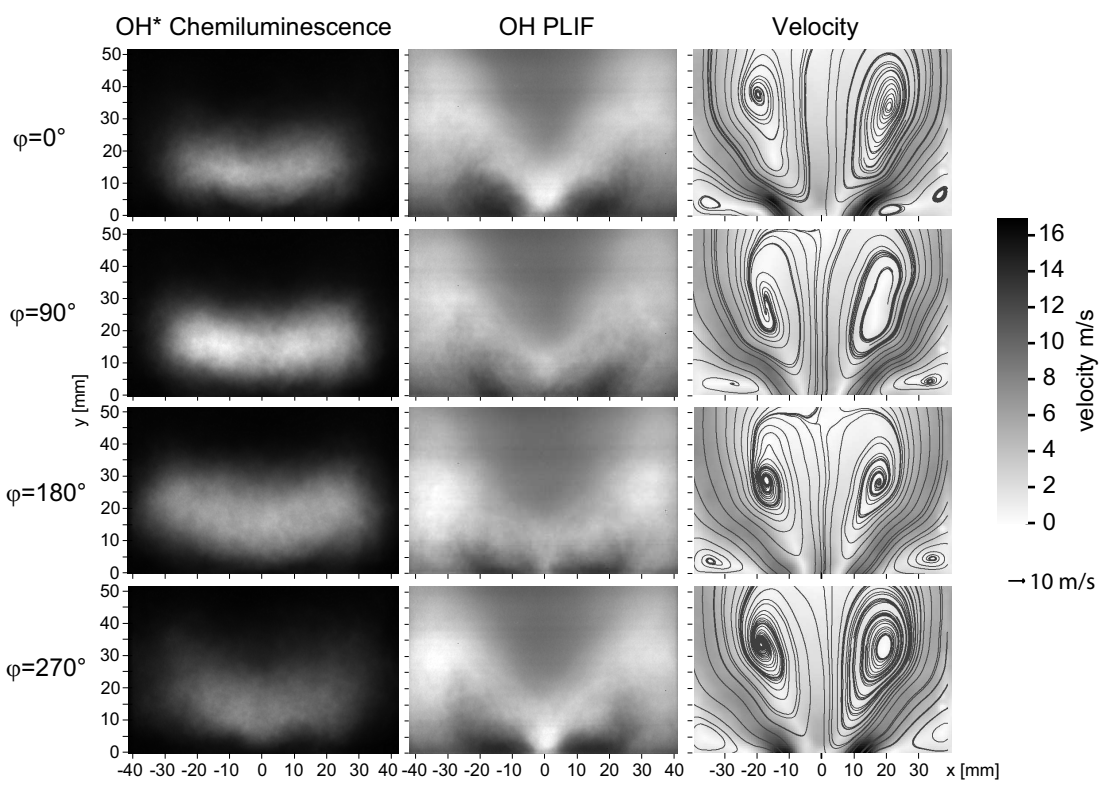

Fig. 3. Phase averages of $\mathrm{OH}^{*}$ chemiluminescence (integrated along line-of-sight), OH-PLIF and velocity in the vertical section. The OH-PLIF images have been corrected for absorption of laser light along the beam path [R. Giezendanner, unpublished data].
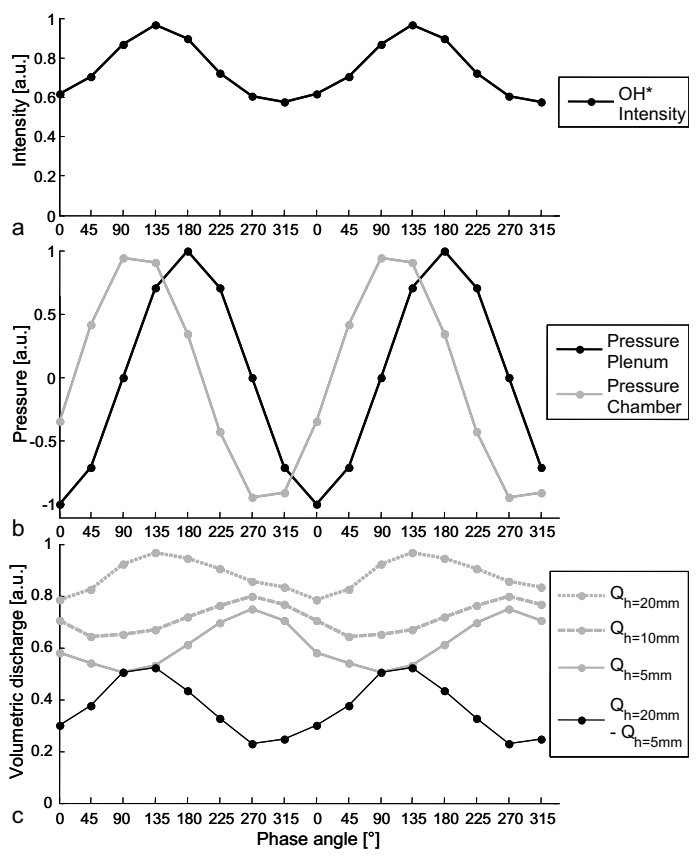

Fig. 4. Phase-dependent averages of (a) $\mathrm{OH}^{*}$-chemiluminesence, (b) normalized pressures and (c) volumetric flow $Q$ through horizontal sections at $y=5,10$ and $20 \mathrm{~mm}$. 
Figure 4 shows phase averaged variations of global heat release, pressure and volumetric discharge $Q$ through different horizontal sections during the oscillation cycle. The values of $Q$ have been obtained from integration of axial velocity $u$ in the horizontal velocity fields. The inflow of unburned gases, roughly represented by $Q$ at $y=5 \mathrm{~mm}$, varies by a factor of $\approx 1.5$ between minimum at $\varphi=90^{\circ}$ and maximum at $\varphi=270^{\circ}$. Global $\mathrm{OH}^{*}$ intensity varies by a similar factor but with a phase-shifted minimum $\left(\varphi=315^{\circ}\right)$ and maximum $\left(\varphi=135^{\circ}\right)$. Pressure in the combustion chamber oscillates roughly in phase with $\mathrm{OH}^{*}$ intensity. Values of $Q$ increase downstream due to thermal expansion in the reaction zone. The increase of $Q$ between $y=5 \mathrm{~mm}$ and $y=20 \mathrm{~mm}$ $\left(Q_{y=20 \mathrm{~mm}^{-}} Q_{y=5 \mathrm{~mm}}\right)$ varies in phase with pressure and $\mathrm{OH}^{*}$ intensity.

\section{Instantaneous structures}

Figure 5a shows an example of instantaneous distributions of velocity and $\mathrm{OH}$ in the vertical section. The velocity field shows the presence of small non-stationary vortices in the inner (ISL) and outer shear layers (OSL). The vortices in the ISL (marked with circles) are ordered in a zig-zag arrangement, which is a strong indication for the formation of a PVC. The corresponding $\mathrm{OH}-\mathrm{PLIF}$ image shows regions without $\mathrm{OH}$ (black) representing unburned gas that has entered from the nozzle below. High levels of $\mathrm{OH}$ (light gray to white) indicate 'young' superequilibrium $\mathrm{OH}$, which is formed in the reaction zones and has a half-life period of $\approx 1 \mathrm{~ms}$ under atmospheric pressure [24]. Medium and low levels of $\mathrm{OH}$ (medium and dark gray) represent burned gas whose $\mathrm{OH}$ concentration has decayed towards equilibrium while it was transported away from the reaction zone. The combination of velocities and $\mathrm{OH}$ shows that the vortices in the ISL (the PVC) and OSL contribute significantly to mixing of hot burned gas into the cold unburned gas, leading to ignition and thereby to stabilization of the flame above the nozzle. The instantaneous velocity field further demonstrates that the time and length scales of recirculation are governed by the unsteady small-scale vortices and not by the comparably much larger scales suggested by the averaged fields shown in fig. 3 .

Figures $5 \mathrm{~b}$ and $5 \mathrm{c}$ show measurements in horizontal sections at $y=5 \mathrm{~mm}$ and $y=10 \mathrm{~mm}$. Generally two distinct large-scale structures of velocity and $\mathrm{OH}$ were found in both transverse sections. The first type, shown in fig. 5b, is characterized by a roughly circular region of inflowing unburned gas, represented by high axial velocity $u$ (red) and absence of $\mathrm{OH}$. The interior of this annulus is filled with high levels of $\mathrm{OH}$ revealing intense chemical reactions in the IRZ. A zone of high negative axial velocities (blue) in the center indicates recirculation of mostly burned gas towards the nozzle. In the outer regions recirculation is slow, and only small areas of superequilibrium $\mathrm{OH}$ are found indicating that reaction is restricted to small isolated regions. The velocity 

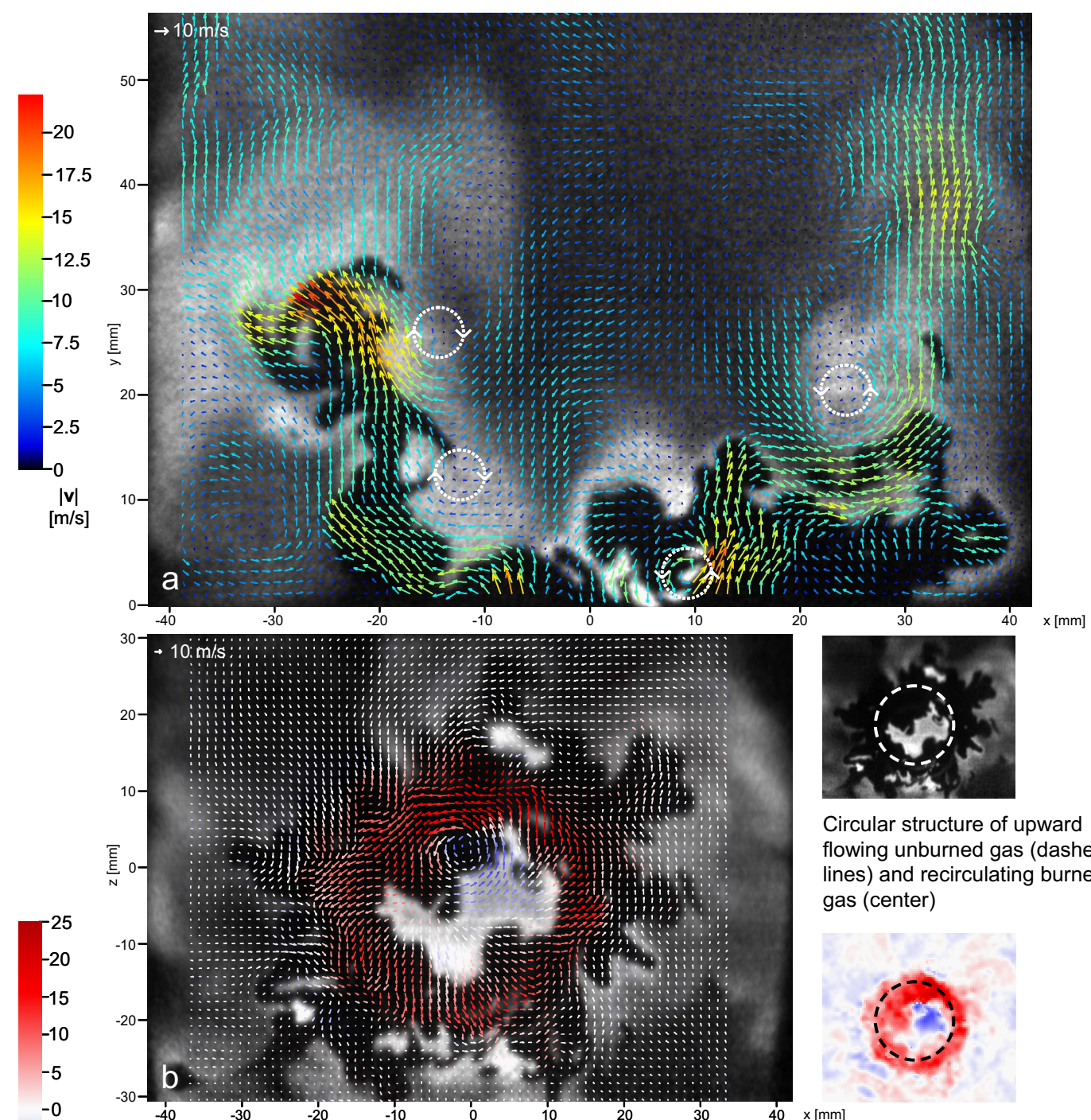

Circular structure of upward flowing unburned gas (dashed lines) and recirculating burned gas (center)

--5
--10
-15
-20
-25

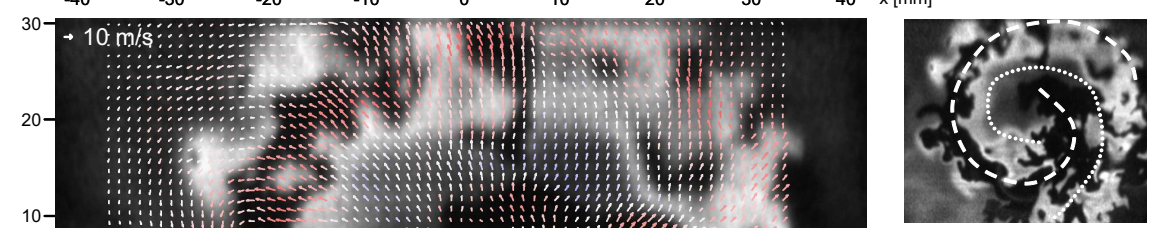

$\mathrm{u}$
$[\mathrm{m} / \mathrm{s}]$

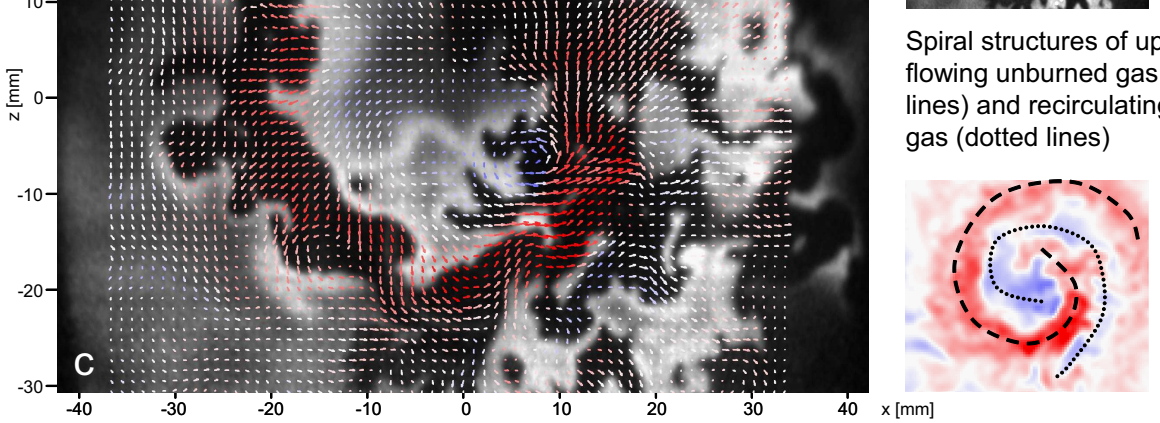

Fig. 5. Simultaneous PIV and OH-PLIF measurements in the vertical section (a) and horizontal sections at $y=5 \mathrm{~mm}(\mathrm{~b})$ and $y=10 \mathrm{~mm}$ (c). Vector colors represent the absolute value of velocity for the vertical section and axial velocity $u$ for horizontal sections. 
field further shows an off-centered axial vortex located on the boundary of the IRZ. This is the typical flow structure of vortex breakdown with a PVC in a transverse plane $[1,8]$.

In a second type of large-scale structures, represented by fig. $5 \mathrm{c}$, the region of positive axial velocity forms a spiral. The area of unburned gas and the reaction zones also have spiral structures. The recirculation zone (blue) consists of an inner region that is connected to the outer parts by a narrow curved zone along the spiral arm. The PVC is represented by an off-centered axial vortex at the inner boundary of the high axial velocities. While the central region features low levels of $\mathrm{OH}$, the areas of high $\mathrm{OH}$ levels indicate that intense chemical reaction takes place along the outer parts of the spiral of unburned gas. Compared to the annular form the spiral shape has a larger boundary between burned and unburned gas and therefore permits a higher global burning rate. While the annular form of positive axial velocity with its distinct inner and outer recirculation zones is a common manifestation of vortex breakdown, the transient spiral recirculation zone observed here has, to our knowledge, not yet been reported.

Similar to the discussion of vortices in the vertical section, the instantaneous horizontal images show that the axial vortex increases mixing of burned and unburned gas in the inner shear layer, and therefore contributes to flame stabilization. The zig-zag ordered vortices in the vertical section and the axial vortex in horizontal section are two aspects of the three-dimensional helical structure of the PVC, which has been shown in several numerical studies of PVCs in confined swirl flames [15-19].

\section{Phase-dependent structural changes}

Figure 6 shows typical structures of velocity and $\mathrm{OH}$ in the horizontal sections at $y=5 \mathrm{~mm}$ and $y=10 \mathrm{~mm}$ for different phase-angles of the thermoacoustic oscillation cycle. At $\varphi=0^{\circ}$ strong inflow of unburned gas takes place (fig. 3) with a mostly annular structure. Global reaction rate and heat release are low (fig. 4a); high levels of $\mathrm{OH}$ in the center indicate that chemical reactions are intense at the boundary of the IRZ, whereas almost no reactions are observed in the ORZ. At $\varphi=90^{\circ}$ the inflow of unburned gas is reduced, global reaction rate has increased to a high level, and the structures of axial velocity and $\mathrm{OH}$ have a spiral form. Zones of intense chemical reactions are mostly found in the inner regions of the spirals $(r \lesssim 25 \mathrm{~mm})$. The reaction zones are highly wrinkled, but the unburned gas as a whole still has a mostly interconnected structure. The inflow of unburned gas remains low and the global reaction rate is still high for $\varphi=180^{\circ}$. The spirals have further expanded in radial direction, and high levels of $\mathrm{OH}$ are found almost over the entire cross-section. Especially at $y=10$ 


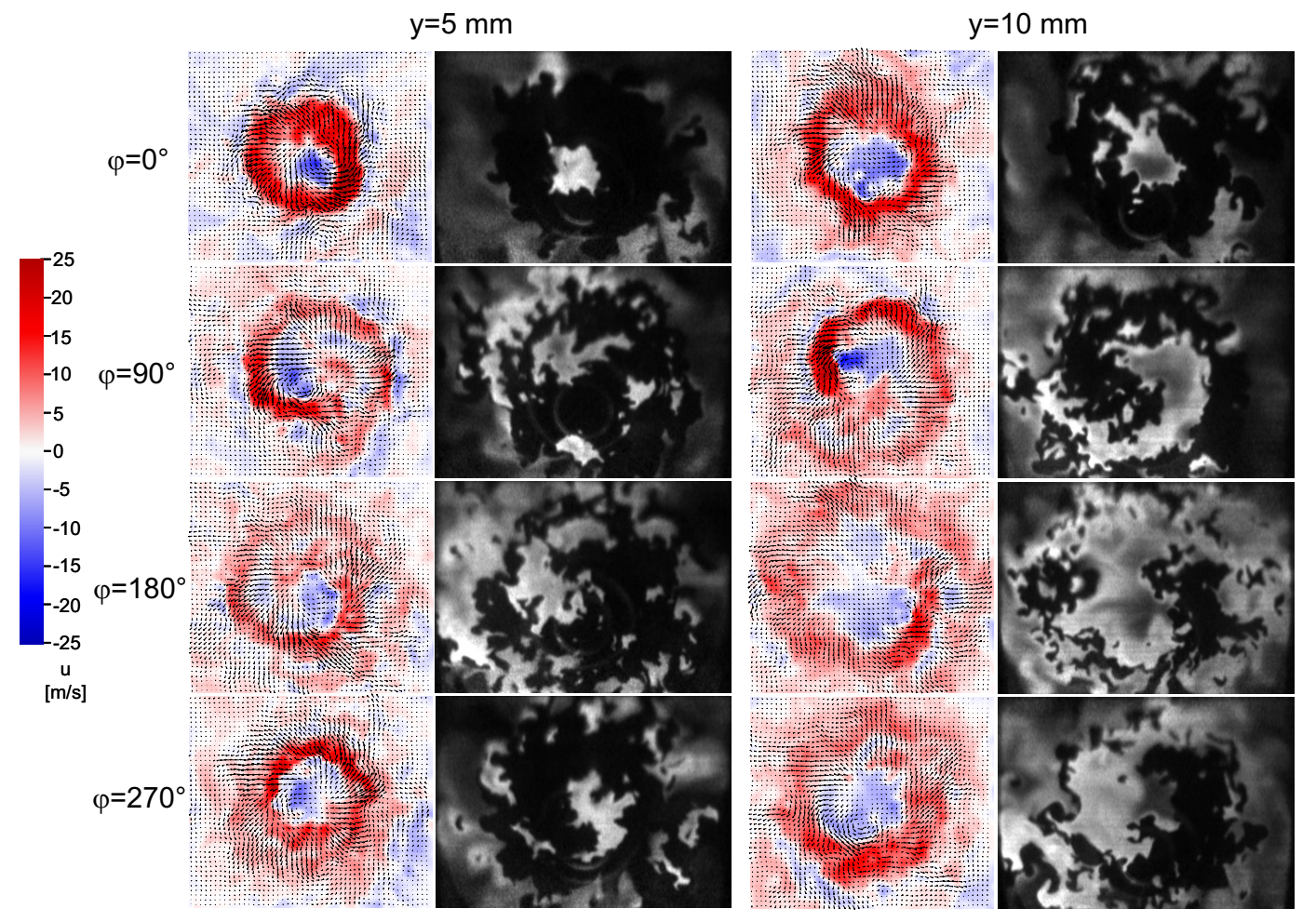

Fig. 6. Simultaneous PIV and OH-PLIF measurements in transverse sections at $y=5$ $\mathrm{mm}$ and $y=10 \mathrm{~mm}$ for different phase-angles $\varphi$.

$\mathrm{mm}$ the amount of unburned gas has further decreased, and the remaining parts are mostly distributed in disconnected regions. At $\varphi=270^{\circ}$ the reaction rate has decreased almost to the minimum, the inflow of unburned gas starts to increase again, and the flow field returns to the annular shape. The phasedependent structural changes show that the appearance of the spiral form is correlated with high rates of chemical reaction and thermal expansion. Effects of fluid dynamics and chemistry might have a mutual positive feedback: On one hand, the spiral form has a larger boundary between burned and unburned gas and therefore favors a higher total reaction rate. On the other hand, thermal expansion resulting from increased reaction rate may induce the breakup of an annular into a spiral form. It seems likely that the off-centered PVC plays a role in the transition from (roughly) circular to spiral symmetry. Detailed insights, however, are limited due to the low temporal resolution of measurements.

It was observed that the azimuthal orientation of the large-scale structures is not correlated with the phase-angle of the thermoacoustic oscillation. As an example, fig. 7a shows 4 additional PLIF images for $\varphi=90^{\circ}$ and $y=10$ $\mathrm{mm}$ where spirals appear with varying orientations. The corresponding phaseaveraged fields of velocity and $\mathrm{OH}$ shown in fig. $7 \mathrm{~b}$ accordingly do not feature a spiral structure but an axial symmetry. It is therefore concluded that the PVC rotates with a frequency different from that of the thermoacoustic instability, and that the two phenomena are not directly correlated. 


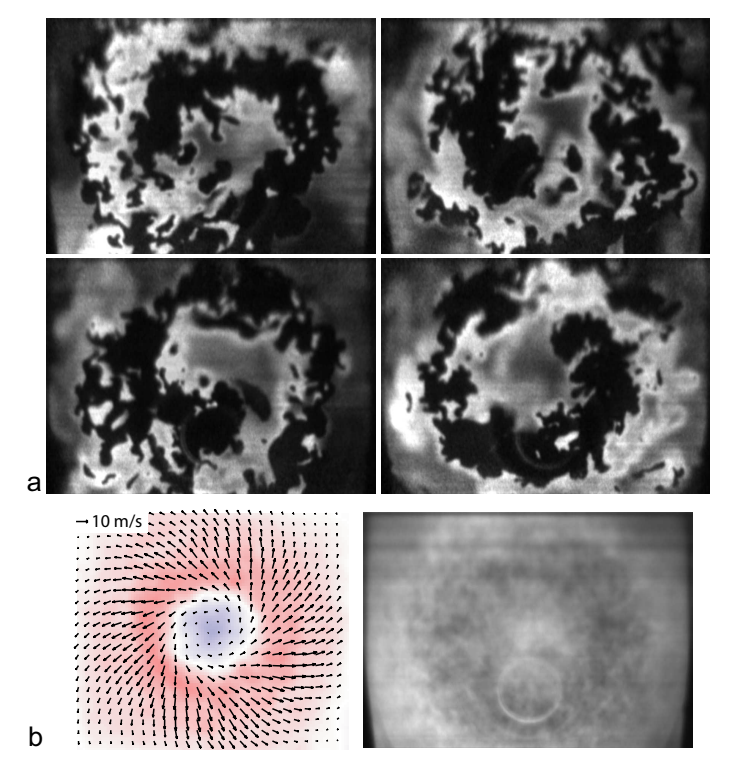

Fig. 7. (a) OH-PLIF images at $y=10 \mathrm{~mm}$ for $\varphi=90^{\circ}$ with different azimuthal orientations and (b) corresponding phase-averages of velocity and $\mathrm{OH}$.

\section{Summary and Conclusions}

Distributions of velocity and $\mathrm{OH}$ were measured simultanously in the central vertical section and three transverse sections of a swirl-stabilized, oscillating flame in a gas turbine model combustor. The averaged flow field with inner and outer recirculation zones was typical of swirl-stabilized flames. The instantaneous measurements showed the presence of a helical vortex (PVC) located in the inner shear layer, which leads to an enhanced mixing of burned and unburned gas and thus to stabilization of the flame. It was found that the large-scale structure of reaction zone and velocity field changes significantly during the cycle of the thermoacoustic oscillation. Two structural types were identified in the transverse sections, an annular and a spiral form. Whereas the first type complies with the classical scenario of vortex breakdown with a PVC, the transient spiral recirculation zone observed here has, to our knowledge, not yet been reported. A phase-resolved analysis showed that the annular form correlates with low, and the spiral form with high rates of global heat release.

A more detailed study of the dynamics of these structures requires timeresolved measurements using $\mathrm{kHz}$ laser diagnostics, which are currently in preparation. Furthermore a numerical study of the flame using LES is under way. 


\section{References}

[1] O. Lucca-Negro, T. O'Doherty, Prog. Energy Combust. Sci. 27 (2001) 431-481.

[2] A. K. Gupta, D. G. Lilley, N. Syred, Swirl flows, Abacus Press, 1984

[3] M. P. Escudier, J. J. Keller, AIAA Journal 23 (1985) 111-116.

[4] K. Midgley, A. Spencer, J. J. McGuirk, J. Eng. Gas Turbines Power 127 (2005) 755-764.

[5] P. M. Anacleto, E. C. Fernandes, M. V. Heitor, S. I. Shtork, Combust. Sci. and Tech. 175 (2003) 1369-1388.

[6] E. C. Fernandes, M. V. Heitor, S. I. Shtork, Exp. Fluids 40 (2006) 177-187.

[7] C. E. Cala, E. C. Fernandes, M. V. Heitor, S. I. Shtork, Exp. Fluids 40 (2006) 267-276.

[8] N. Syred, Prog. Energy Combust. Sci. 32 (2006) 93-161.

[9] K.-U. Schildmacher, R. Koch, J. Eng. Gas Turbines Power 127 (2005) 295-300.

[10] N. Syred, W. Fick, T. O’Doherty, A. J. Griffiths, Combust. Sci. and Tech. 125 (1997) 139-157.

[11] G. Li, E. J. Gutmark, AIAA 42nd (2004) 133.

[12] M. Garcia-Villalba, J. Fröhlich, W. Rodi, Phys. Fluids 18 (2006) 055103.

[13] S. Wang, V. Yang, G. Hsiao, S.-Y. Hsieh, H. C. Mongia, J. Fluid Mech. 583 (2007) 99122.

[14] A. Widenhorn, B. Noll, M. Stöhr, M. Aigner, in: S.-H. Peng, W. Haase (Eds.), Advances in Hybrid RANS-LES Modelling, Springer, Berlin Heidelberg, 2007, pp. 152-161.

[15] L. Selle, G. Lartigue, T. Poinsot, R. Koch, K.-U. Schildmacher, W. Krebs, B. Prade, P. Kaufmann, D. Veynante, Combust. Flame 137 (2004) 489505.

[16] S. Roux, G. Lartiguea, T. Poinsot, U. Meier, C. Berat, Combust. Flame 141 (2005) 4054.

[17] Y. Huang, V. Yang, Proc. Combust. Inst. 30 (2005) 17751782.

[18] L. Selle, L. Benoit, T. Poinsot, F. Nicoud, W. Krebs, Combust. Flame 145 (2006) 194-205.

[19] C. Duwig, L. Fuchs, Phys. Fluids 19 (2007) 075103.

[20] M. Freitag, J. Janicka, Proc. Combust. Inst. 31 (2007) 1477-1485.

[21] A. X. Sengissen, A. V. Giauque, G. S. Staffelbach, M. Porta, W. Krebs, P. Kaufmann, T. J. Poinsot, Proc. Combust. Inst. 31 (2007) 1729-1736. 
[22] O. Stein, A. Kempf, Proc. Combust. Inst. 31 (2007) 1755-1763.

[23] C. Fureby, F. F. Grinstein, G. Li, E. J. Gutmark, Proc. Combust. Inst. 31 (2007) 3107-3114.

[24] R. Sadanandan, M. Stöhr, W. Meier, Appl. Phys. B 90 (2008) 609-618.

[25] R. Giezendanner, O. Keck, P. Weigand, W. Meier, U. Meier, W.Stricker, M. Aigner, Combust. Sci. and Tech. 175 (2003) 721-741.

[26] X. R. Duan, W. Meier, P. Weigand, B. Lehmann, Appl. Phys. B 80 (2005) 389-396.

[27] W. Meier, X. R. Duan, P. Weigand, Proc. Combust. Inst. 30 (2005) 835-842.

[28] P. Weigand, W. Meier , X. R. Duan, W. Stricker, M. Aigner, Combust. Flame 144 (2006) 205224.

[29] W. Meier , X. R. Duan, P. Weigand, Combust. Flame 144 (2006) 225236. 